\title{
Effects of dexmedetomidine on post-operative recovery and mental status in patients receiving robotic-assisted thoracic surgery
}

\author{
Li-Yun Zhang, Yu-Hao Zhang, Jie Shen, Yan Luo \\ Department of Anesthesiology, Ruijin Hospital, Shanghai Jiaotong University School of Medicine, Shanghai 200025, China \\ Contributions: (I) Conception and design: LY Zhang, Y Luo; (II) Administrative support: Y Luo; (III) Provision of study materials or patients: LY \\ Zhang; (IV) Collection and assembly of data: LY Zhang, YH Zhang, J Shen; (V) Data analysis and interpretation: LY Zhang, YH Zhang; (VI) \\ Manuscript writing: All authors; (VII) Final approval of manuscript: All authors. \\ Correspondence to: Yan Luo. Department of Anesthesiology, Ruijin Hospital, Shanghai Jiaotong University School of Medicine, Shanghai 200025, \\ China. Email: 18917762576@163.com.
}

Background: This study aimed to investigate the effects of dexmedetomidine on the post-operative recovery and mental status in patients receiving robotic-assisted thoracic surgery (RATS).

Methods: One hundred patients who received selective RATS under general anesthesia were recruited and assigned into control group (C) and dexmedetomidine group (D). The anesthesia induction and maintenance were consistent between groups. Midazolam, sufentanil, propofol and rocuronium were intravenously injected for anesthesia induction, followed by mechanical ventilation after endotracheal intubation. Sevoflurane inhalation at a minimum alveolar concentration (MAC) of 0.5 was administered, propofol and remifentanil were intravenously injected to maintain the bispectral index (BIS) at 40-60, and rocuronium was intravenously injected once every $30 \mathrm{~min}$. In the D group, dexmedetomidine was intravenously injected after endotracheal intubation, and then it was injected before the end of surgery. In the $\mathrm{C}$ group, normal saline of equal volume was injected. The hemodynamic parameters, blood loss, urine volume, time of surgery, time of anesthesia, total dose of propofol, time of thoracic tube indwelling, hospital stay and pulmonary complications were recorded; blood gas analysis was performed after extubation; the QoR-15 and minimental state examination (MMSE) questionnaires were employed for the assessment of mental status at 1 and 3 days after surgery.

Results: The mean arterial pressure (MAP), heart rate (HR) and brain oxygenation were similar between groups at different time points $(\mathrm{P}>0.05)$. There were no significant differences in the operation time, time of anesthesia and intra-operative urine volume between groups. As compared to the $\mathrm{C}$ group, the blood loss and dose of propofol reduced significantly $(\mathrm{P}<0.05)$. After extubation, the respiratory frequency reduced and $\mathrm{PaO} 2$ increased markedly $(\mathrm{P}<0.05)$. After surgery, the time of thoracic tube indwelling and hospital stay reduced dramatically in the $\mathrm{D}$ group as compared to the $\mathrm{C}$ group $(\mathrm{P}<0.05)$. The QoR-15 score and MMSE score in the $\mathrm{D}$ group were markedly higher than in the $\mathrm{C}$ group $(\mathrm{P}<0.05)$.

Conclusions: Dexmedetomidine can improve the post-operative recovery and mental status after RATS.

Keywords: Dexmedetomidine; robotic-assisted surgery; thoracic surgery; post-operative recovery; post-operative mental status

Submitted Apr 05, 2019. Accepted for publication Aug 08, 2019.

doi: 10.21037/apm.2019.08.09

View this article at: http://dx.doi.org/10.21037/apm.2019.08.09 


\section{Introduction}

Robotic-assisted thoracic surgery (RATS) as a new surgical technique offers advantages of improved three-dimensional visualization and increased degrees of freedom. In recent years, RATS has been widely applied for thoracic surgery. As compared to the traditional thoracic surgery, RATS has the advantages of minimal invasion, rapid post-operative recovery and convenient lymph node dissection (1), which reduce the surgical difficulty and make the surgery more comfortable for the surgeons. However, patients may still develop acute or chronic pain of different degrees after RATS, which is closely related to the post-operative complications and mortality (2). Available studies have revealed that about $40 \%$ of lung cancer patients experience mental and emotional changes of different extents after surgery, which is slightly higher than that of patients with other caners (3).

Dexmedetomidine is a highly selective $\alpha_{2}$ adrenergic receptor agonist and can act at the peripheral and central levels to exert anti-inflammatory $(4,5)$, analgesic $(6,7)$ and sedative (8) effects via inhibiting catecholamine release dependent of presynaptic membrane receptor; it can activate postsynaptic membrane receptor to inhibit the sympathetic activity, lowering blood pressure and heart rate (HR) and reducing the dose of opioids $(7,9,10)$. This can help to improve the hemodynamic stability during the operation and to reduce the incidence of myocardial ischemia. In addition, dexmedetomidine can inhibit $\alpha_{2}$ receptor in the nucleus coeruleus, which reduces the release of substance $\mathrm{P}$ and other harmful peptides, suppressing the transduction of pain-related signals and resulting in the sedation and anxiety relief $(11,12)$. Thus, dexmedetomidine is frequently used as an adjutant during the general anesthesia in clinical practice (13).

However, little is known about the influence of dexmedetomidine in patients receiving RATS, and the perioperative outcomes should be further confirmed. This study aimed to investigate the effects of dexmedetomidine on the post-operative recovery and mental status in patients receiving RATS under general anesthesia, which may provide evidence for future clinical use of dexmedetomidine and the assessment of prognosis.

\section{Methods}

This study was approved by the Ethics Committee of Ruijin Hospital (No. 2018-211). A total of 100 patients receiving selective RATS under general anesthesia were recruited into the present study. This sample size was calculated with the MedSci software (MedSci Sample Size tools, MSST). The age ranged from 20 years to 70 years; the body mass index (BMI) was $<30 \mathrm{~kg} / \mathrm{m}^{2}$; the ASA physical status was I or II; there were no severe bradycardia ( $<45$ beat $/ \mathrm{min}$ ), no severe liver and kidney dysfunction, and no mental diseases. Patients were randomly assigned into 2 groups ( $\mathrm{n}=50$ per group): normal saline control group $(\mathrm{C})$ and dexmedetomidine group (D).

Patients received food deprivation for $8 \mathrm{~h}$ and water deprivation for $4 \mathrm{~h}$, and drugs were not used before surgery. The operation room temperature was maintained at $23-25^{\circ} \mathrm{C}$. After being transferred into the operation room, patients received oxygen inhalation at $3 \mathrm{~L} / \mathrm{min}$ via a mask, and the $\mathrm{HR}$, blood oxygen saturation $\left(\mathrm{SpO}_{2}\right)$, electrocardiogram (ECG) and blood pressure (BP) were routinely monitored. After focal anesthesia, the radial artery was punctured, and a venous access was established for invasive BP monitoring and bispectral index (BIS) monitoring with the NarcotrendCompact monitor (MT Monitor Technik GmbH\&Co. $\mathrm{KG}$, Germany). Then, rapid sequential induction was employed for the anesthesia induction. Midazolam (Lot No: 20180102; Yichang Renfu Pharmaceutical Co., Ltd., China) at $0.4 \mu \mathrm{g} / \mathrm{kg}$, propofol (Lot No: NJ807; Corden Pharma S.P.A; Italy) at $1.5-2.0 \mathrm{mg} / \mathrm{kg}$ and rocuronium (Lot No: 171002; Zhejiang Xianju Pharmaceutical Co., Ltd., China) at $0.6 \mathrm{mg} / \mathrm{kg}$ were sequentially injected intravenously. After intubation of double-lumen endobronchial tube, bronchoscopy was performed to confirm the location of endotracheal tube. Then, one-lung ventilation (OLV) was implemented. Mechanical ventilation was performed with tidal volume at $6-8 \mathrm{~mL} / \mathrm{kg}$, ventilation frequency of 12-14 breaths/min, inspiratory-to-expiratory ratio at $1: 1.5$, oxygen flow rate at $1.5 \mathrm{~L} / \mathrm{min}$ and oxygen concentration at $100 \%$. The end-tidal carbon dioxide partial pressure $\left(\mathrm{P}_{\mathrm{ET}} \mathrm{CO}_{2}\right)$ was maintained at $35-45 \mathrm{mmHg}$ (1 $\mathrm{mmHg}=0.133 \mathrm{kPa})$. Anesthesia maintenance: $1.5-2.5 \%$ sevoflurane (Lot No: S045C729; Baxter International Inc, USA) inhalation was done with minimum alveolar concentration (MAC) at 0.5 , and rocuronium $(5 \mathrm{mg})$ was intravenously injected once every $30 \mathrm{~min}$. The intravenous injection was done with a transfusion station (B. Braun Medical Inc., Germany). The target controlled infusion (TCI) of propofol at $1-1.5 \mu \mathrm{g} / \mathrm{mL}$ and remifentanil (Lot No: 6171206; Yichang Renfu Pharmaceutical Co., Ltd.) at $0.05-0.15 \mu \mathrm{g} / \mathrm{kg} / \mathrm{min}$ were employed to maintain the BIS at 40-60. In the D group, dexmedetomidine 
Table 1 General characteristics of patients in two groups ( $\mathrm{n}=50$ per group)

\begin{tabular}{lcccccc}
\hline Group & Age (years) & Gender (M/F) & Height $(\mathrm{cm})$ & Body weight $(\mathrm{kg})$ & BMl $\left(\mathrm{kg} \bullet \mathrm{m}^{-2}\right)$ & ASA grade $(\mathrm{n}, \mathrm{I} / \mathrm{II})$ \\
\hline C & $58 \pm 12$ & $24 / 26$ & $165.7 \pm 6.1$ & $62.2 \pm 7.3$ & $22.6 \pm 2.1$ & $6 / 44$ \\
D & $56 \pm 11$ & $29 / 21$ & $167.4 \pm 8.7$ & $65.1 \pm 12.5$ & $23.1 \pm 3.3$ & $4 / 46$ \\
\hline
\end{tabular}

C, control group; D, dexmedetomidine group; M, male; F, female; BMI, body mass index; ASA, American Society of Anesthesiologists.

Table 2 MAP, HR and brain oxygenation at different time points in two groups ( $\mathrm{n}=50$ per group)

\begin{tabular}{lccccccc}
\hline Monitoring index & Group & T0 & OLV-10 & OLV-20 & OLV-30 & OLV-40 & TLV-15 \\
\hline MAP (mmHg) & C & $83 \pm 10$ & $77 \pm 6$ & $76 \pm 8$ & $76 \pm 9$ & $75 \pm 8$ & $83 \pm 8$ \\
& D & $79 \pm 11$ & $78 \pm 13$ & $79 \pm 10$ & $75 \pm 8$ & $73 \pm 9$ & $70 \pm 8$ \\
HR (beat/min) & C & $67 \pm 8$ & $69 \pm 12$ & $67 \pm 9$ & $70 \pm 8$ & $73 \pm 9$ & $73 \pm 9$ \\
Brain oxygenation (\%, L/R) & D & $67 \pm 11$ & $66 \pm 7$ & $68 \pm 8$ & $68 \pm 9$ & $70 \pm 9$ & $75 \pm 5 / 71 \pm 7$ \\
& C & $74 \pm 7 / 71 \pm 6$ & $72 \pm 7 / 69 \pm 7$ & $72 \pm 6 / 68 \pm 9$ & $73 \pm 7 / 69 \pm 9$ & $76 \pm 6 / 73 \pm 8$ \\
& D & $76 \pm 7 / 72 \pm 8$ & $72 \pm 6 / 70 \pm 9$ & $72 \pm 7 / 69 \pm 10$ & $72 \pm 5 / 69 \pm 8$ & $73 \pm 7 / 69 \pm 9$ & $75 \pm 8 / 72 \pm 10$ \\
\hline
\end{tabular}

MAP, mean arterial pressure; HR, heart rate; OLV, one-lung ventilation; TLV, two-lung ventilation; C, control group; D, dexmedetomidine group.

(Lot No: 1712261; Sichuan Guorui Pharmaceutical Co., Ltd., China) was injected at $1 \mu \mathrm{g} / \mathrm{kg} 10 \mathrm{~min}$ after endotracheal intubation and then at $0.4 \mu \mathrm{g} / \mathrm{kg} / \mathrm{h}$ until 30 min before surgery. In the $\mathrm{C}$ group, normal saline of equal volume was injected. Ephedrine $(5 \mathrm{mg}$ ) (Lot No: 180304; Northeast Pharm Co., Ltd, China) was injected when the mean arterial pressure (MAP) was lower than $20 \%$ of baseline level; atropine $(0.5 \mathrm{mg}$ ) (Lot No: 1803311; Tianjing Jinyao Pharmaceutical Co., Ltd., China) was administered when the HR was lower than 55 beats $/ \mathrm{min}$. After being transferred into operation room, lactated Ringer's solution and succinylated gelatin injection were injected at $8-10 \mathrm{~mL} / \mathrm{kg} / \mathrm{h}$ after heating via a HOTLINE blood and solution heater (Shanghai Lanfeng Medical Equipment Co., Ltd., China).

On transferring into operation room (T0), $10 \mathrm{~min}$ after OLV (OLV-10), 20 min after OLV (OLV-20), 30 min after OLV (OLV-30), $40 \mathrm{~min}$ after OLV (OLV-40), and $15 \mathrm{~min}$ after two-lung ventilation (TLV-15), the MAP, HR, brain oxygenation, intra-operative blood loss, urine volume, time of operation, time of anesthesia, and total dose of propofol were recorded. After extubation, the blood gas analysis was done to record $\mathrm{PaO}_{2}$ and $\mathrm{PaCO}_{2}$. In addition, the time of thoracic tube indwelling, hospital stay and pulmonary complications (such as atelectasis, pneumonia and air leak) were also recorded for further analysis. The QoR-15 and
Mini-Mental State Examination (MMSE) questionnaires were employed for the assessment of quality of recovery and mental state, respectively, before surgery, and 1 (POD1) and 3 (POD3) after surgery.

Statistical analysis was done with SPSS version 20.0. Quantitative data are expressed as means \pm standard deviation $(\overline{\mathrm{x}} \pm \mathrm{s})$. One-way analysis of variance was employed for intergroup comparisons. Qualitative data were compared with Chi square test. A value of $\mathrm{P}<0.05$ was considered statistically significant.

\section{Results}

In this study, a total 100 patients were recruited, and the general characteristics were comparable at baseline between the $\mathrm{C}$ group and the $\mathrm{D}$ group $(\mathrm{P}>0.05)$ (Table 1).

At different time points, there were no marked differences in the MAP, HR and brain oxygenation between two groups $(\mathrm{P}>0.05)$ (Table 2). In addition, the time of operation, time of anesthesia and intra-operative urine volume were comparable between groups $(\mathrm{P}>0.05)$. When compared with the $\mathrm{C}$ group, the intra-operative blood loss and total dose of propofol reduced significantly in the $\mathrm{D}$ group $(\mathrm{P}<0.05)$ (Table 3).

After extubation, the $\mathrm{PaO}_{2}$ in the $\mathrm{D}$ group was significantly higher than in the $\mathrm{C}$ group $(\mathrm{P}<0.05)$, the 
Table 3 Intra-operative characteristics of patients in two groups ( $\mathrm{n}=50$ per group)

\begin{tabular}{lccccc}
\hline Group & $\begin{array}{c}\text { Time of surgery } \\
(\mathrm{min})\end{array}$ & $\begin{array}{c}\text { Time of anesthesia } \\
(\mathrm{min})\end{array}$ & $\begin{array}{c}\text { Intra-operative blood } \\
\text { loss }(\mathrm{mL})\end{array}$ & $\begin{array}{c}\text { Intra-operative urine volume } \\
(\mathrm{mL})\end{array}$ & $\begin{array}{c}\text { Total dose of propofol } \\
(\mathrm{mg})\end{array}$ \\
\hline C & $159 \pm 88$ & $201 \pm 85$ & $149 \pm 67$ & $552 \pm 309$ & $723 \pm 378$ \\
D & $153 \pm 75$ & $205 \pm 82$ & $120 \pm 52^{*}$ & $581 \pm 391$ & $512 \pm 347^{\star}$ \\
\hline
\end{tabular}

*, $\mathrm{P}<0.05$ vs. C group. C, control group; D, dexmedetomidine group.

Table 4 Respiratory frequency, $\mathrm{PaO}_{2}$ and $\mathrm{PaCO}_{2}$ after extubation in two groups ( $\mathrm{n}=50$ per group)

\begin{tabular}{lccc}
\hline Group & Respiratory frequency (breath/min) & $\mathrm{PaO}_{2}(\mathrm{mmHg})$ & $\mathrm{PaCO}(\mathrm{mmHg})$ \\
\hline C & $20 \pm 3$ & $80.4 \pm 5.4$ & $40.0 \pm 2.6$ \\
D & $17 \pm 3^{\star}$ & $89.0 \pm 12.9^{*}$ & $40.2 \pm 3.4$ \\
\hline
\end{tabular}

${ }^{*}, \mathrm{P}<0.05$ vs. C group. C, control group; D, dexmedetomidine group.

Table 5 Post-operative complications and hospital stay in two groups ( $\mathrm{n}=50$ per group)

\begin{tabular}{lccc}
\hline Group & Pulmonary complications $(\mathrm{n})$ & Time of thoracic tube indwelling $(\mathrm{d})$ & Post-operative hospital stay $(\mathrm{d})$ \\
\hline C & 8 & $4.4 \pm 2.7$ & $6.0 \pm 3.1$ \\
$\mathrm{D}$ & 6 & $3.2 \pm 1.8^{*}$ & $4.6 \pm 2.2^{*}$ \\
\hline
\end{tabular}

${ }^{*}, \mathrm{P}<0.05$ vs. C group. C, control group; D, dexmedetomidine group.

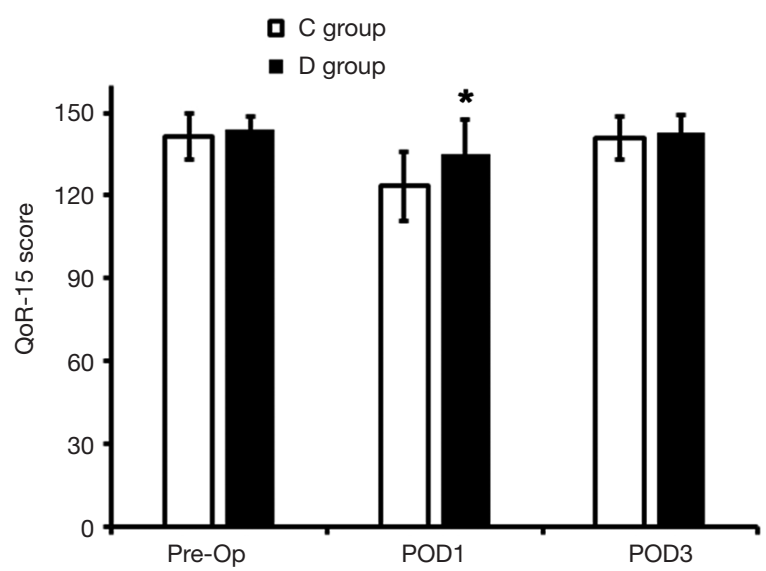

Figure 1 QoR-15 score of patients in two groups. *, $\mathrm{P}<0.05$ vs. $\mathrm{C}$ group. C group, control group; D group, dexmedetomidine group; POD, post-operative day.

respiratory frequency in the $\mathrm{D}$ group was markedly lower than in the $\mathrm{C}$ group $(\mathrm{P}<0.05)$, but the $\mathrm{PaCO}_{2}$ was similar between groups $(\mathrm{P}>0.05)$ (Table 4$)$.

There were no significant differences in the postoperative complications between groups ( $\mathrm{P}>0.05$; Table 5). The time of thoracic tube indwelling and post-operative

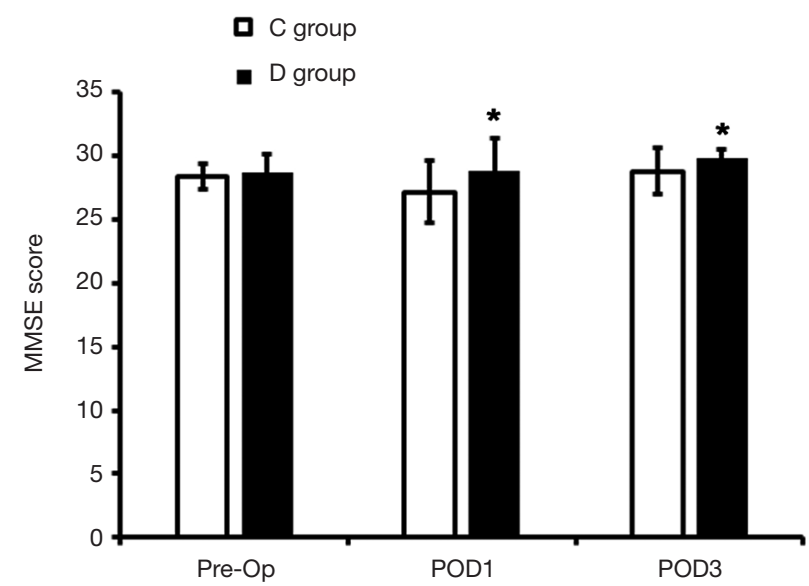

Figure 2 MMSE score of patients in two groups. * $\mathrm{P}<0.05$ vs. $\mathrm{C}$ group. C group, control group; D group, dexmedetomidine group; MMSE, mini-mental state examination; POD, post-operative day.

hospital stay in the $\mathrm{D}$ group reduced significantly as compared to the $\mathrm{C}$ group $(\mathrm{P}<0.05)$.

At POD1, the QoR-15 score and MMSE score in the D group were significantly higher than in the $\mathrm{C}$ group $(\mathrm{P}<0.05$; Figures 1,2); at POD3, the MMSE score in D group was markedly higher than in the $\mathrm{C}$ group $(\mathrm{P}<0.05)$, but the 
QoR-15 score was comparable between groups $(\mathrm{P}>0.05)$.

\section{Discussion}

Dexmedetomidine is a highly selective $\alpha_{2}$ adrenergic receptor agonist and can inhibit the norepinephrine release and sympathetic activity, which reduce the required dose of anesthetics (14). In the present study, our results showed the hemodynamic parameters remained stable in two groups as demonstrated by the comparable MAP, HR and brain oxygenation between two groups. However, the total dose of propofol in the D group was significantly lower than in the $\mathrm{C}$ group, suggesting that dexmedetomidine as an adjuvant for general anesthesia can reduce the required dose of anesthetics.

In the present study, the respiratory frequency reduced in the D group after extubation as compared to the C group, the oxygenation was improved, and the respiratory function in the $\mathrm{D}$ group was also superior to that in the $\mathrm{C}$ group. There is evidence $(15,16)$ showing that the respiratory function is closely related to the severity of pain after thoracic surgery. The incision pain may inhibit the breath and cough of patients, which leads to ineffective ventilation and is also harmful for the sputum expectoration, affecting the post-operative recovery of lung function. Thus, on one hand, dexmedetomidine can modulate the post-operative pain to improve the post-operative oxygenation, and on the other hand, it can reduce the required dose of opioids in the peri-operative period, reducing the inhibitory effect of opioids.

QoR-15 is a questionnaire based on the pain, anxiety and comfort, and it has been widely used for the assessment of post-operative recovery $(17)$. Studies $(7,9,18)$ have shown that dexmedetomidine can attenuate the post-operative anxiety and improve the pain and satisfaction after surgery. Although the half-life of dexmedetomidine is $2-2.5 \mathrm{~h}$ (19), the use of dexmedetomidine in the present study increased the QoR-15 score, suggesting the improvement of postoperative recovery, but the specific mechanism is unclear. Ge et al. (18) found dexmedetomidine can not only act on the $\alpha_{2}$ adrenergic receptor, but also prolong the analgesic effect of other drugs, which indirectly improves the analgesia, prolong the analgesic effect, improves the post-operative comfort and therefore facilitates the postoperative recovery.

MMSE can be used to comprehensively, accurately and rapidly assess the intelligence and cognitive function (20). In the present study, the MMSE score at POD1 and
POD3 in the D group was significantly higher than in the $\mathrm{C}$ group, suggesting the neuroprotection. Hoffman et al. (21) for the first time reported the neuroprotective effect of dexmedetomidine, but the specific mechanism is still unclear. Animal studies $(22,23)$ have revealed that the neuroprotection of dexmedetomidine may be related to following factors: dexmedetomidine can reduce nitric oxide (NO) and tumor necrosis factor- $\alpha(\mathrm{TNF}-\alpha)$ and increase superoxide dismutase (SOD) to exert neuroprotective effect; dexmedetomidine can inhibit catecholamine release in the brain and mitigate vascular spasm; dexmedetomidine can inhibit inflammation via reducing some blood inflammatory factors, which attenuates stress and thereafter exerts neuroprotective effect (24).

The hospital stay after RATS is affected by the age, physical status and post-operative lung function (25). There is evidence showing that dexmedetomidine can attenuate OLV induced inflammation in the lung during thoracic surgery, which is helpful for the improvement of postoperative outcomes. Jiang et al. (26) found dexmedetomidine could reduce monocyte chemoattractant protein 1 (MCP-1) expression to attenuate lung ischemia/reperfusion injury. Wu et al. (27) found dexmedetomidine was able to inhibit the neutrophil aggregation and mitigate the adverse response to OLV. Mental status besides environmental and clinical characteristics also affects the hospital stay (28). In the present study, there were no significant differences in the general characteristics and post-operative pulmonary complications between groups. However, the QoR-15 score and MMSE score in the D group after surgery were significantly higher than in the $\mathrm{C}$ group, suggesting the better post-operative recovery and better mental status in the $\mathrm{D}$ group. This may be related to the reduced hospital stay. Although information is lacking about the long-term effect of dexmedetomidine, the study of Myles et al. (29) showed the poor post-operative recovery was predictive for poor post-operative quality of life. Thus, the use of dexmedetomidine during RATS may facilitate the postoperative recovery and improve the mental status, which are beneficial for the post-operative improvement of quality of life, satisfaction and comfort.

\section{Conclusions}

Taken together, the use of dexmedetomidine during RATS can reduce the required dose of anesthetics, shorten the hospital stay and improve the post-operative recovery and mental status. 


\section{Acknowledgments}

None.

\section{Footnote}

Conflicts of Interest: The authors have no conflicts of interest to declare.

Ethical Statement: The authors are accountable for all aspects of the work in ensuring that questions related to the accuracy or integrity of any part of the work are appropriately investigated and resolved. This study was approved by the Ethics Committee of Ruijin Hospital (No. 2018-211). Informed consent was obtained from each patient or relatives before surgery.

\section{References}

1. Song Y, Shim JK, Song JW, et al. Dexmedetomidine added to an opioid-based analgesic regimen for the prevention of postoperative nausea and vomiting in highly susceptible patients: A randomised controlled trial. Eur J Anaesthesiol 2016;33:75-83.

2. Esme H, Apiliogullari B, Duran FM, et al. Comparison between intermittent intravenous analgesia and intermittent paravertebral subpleural analgesia for pain relief after thoracotomy. Eur J Cardiothorac Surg 2012;41:10-3.

3. Zabora J, BrintzenhofeSzoc K, Curbow B, et al. The prevalence of psychological distress by cancer site. Psycho-Oncology 2001;10:19-28.

4. Ueki M, Kawasaki T, Habe K, et al. The effects of dexmedetomidine on inflammatory mediators after cardiopulmonary bypass. Anaesthesia 2014;69:693-700.

5. Xiang H, Hu B, Li Z, et al. Dexmedetomidine controls systemic cytokine levels through the cholinergic antiinflammatory pathway. Inflammation 2014;37:1763-70.

6. Rajan S, Hutcherson MT, Sessler DI, et al. The Effects of Dexmedetomidine and Remifentanil on Hemodynamic Stability and Analgesic Requirement After Craniotomy: A Randomized Controlled Trial. J Neurosurg Anesthesiol 2016;28:282-90.

7. Ren C, Chi M, Zhang Y, et al. Dexmedetomidine in Postoperative Analgesia in Patients Undergoing Hysterectomy: A CONSORT-Prospective, Randomized, Controlled Trial. Medicine (Baltimore) 2015;94:e1348.

8. Ebert TJ, Hall JE, Barney JA, et al. The effects of increasing plasma concentrations of dexmedetomidine in humans. Anesthesiology 2000;93:382-94.

9. Blaudszun G, Lysakowski C, Elia N, et al. Effect of perioperative systemic alpha2 agonists on postoperative morphine consumption and pain intensity: systematic review and meta-analysis of randomized controlled trials. Anesthesiology 2012;116:1312-22.

10. Lin TF, Yeh YC, Lin FS, et al. Effect of combining dexmedetomidine and morphine for intravenous patientcontrolled analgesia. Br J Anaesth 2009;102:117-22.

11. Biswas S, Das RK, Mukherjee G, et al. Dexmedetomidine an adjuvant to levobupivacaine in supraclavicular brachial plexus block: a randomized double blind prospective study. Ethiop J Health Sci 2014;24:203-8.

12. Mohta M, Kalra B, Sethi AK, et al. Efficacy of dexmedetomidine as an adjuvant in paravertebral block in breast cancer surgery. J Anesth 2016;30:252-60.

13. Ji F, Li Z, Nguyen H, et al. Perioperative dexmedetomidine improves outcomes of cardiac surgery. Circulation 2013;127:1576-84.

14. Gao J, Zhang Y, Yang J, et al. Sedative and analgesicsparing effects of dexmedetomidine in patients undergoing general anaesthesia. Shanghai Med 2010;33:525-7.

15. Bauer C, Hentz JG, Ducrocq X, et al. Lung function after lobectomy: a randomized, double-blinded trial comparing thoracic epidural ropivacaine/sufentanil and intravenous morphine for patient-controlled analgesia. Anesth Analg 2007;105:238-44.

16. Ried M, Schilling C, Potzger T, et al. Prospective, comparative study of the On-Q(R) PainBuster(R) postoperative pain relief system and thoracic epidural analgesia after thoracic surgery. J Cardiothorac Vasc Anesth 2014;28:973-8.

17. Stark PA, Myles PS, Burke JA. Development and psychometric evaluation of a postoperative quality of recovery score: the QoR-15. Anesthesiology 2013;118:1332-40.

18. Ge DJ, Qi B, Tang G, et al. Intraoperative Dexmedetomidine Promotes Postoperative Analgesia and Recovery in Patients after Abdominal Colectomy: A CONSORT-Prospective, Randomized, Controlled Clinical Trial. Medicine (Baltimore) 2015;94:e1727.

19. Scheinin H, Aantaa R, Anttila M, et al. Reversal of the sedative and sympatholytic effects of dexmedetomidine with a specific alpha2-adrenoceptor antagonist atipamezole: a pharmacodynamic and kinetic study in healthy volunteers. Anesthesiology 1998;89:574-84.

20. Folstein MF, Folstein SE, McHugh PR. "Mini-mental 
state". A practical method for grading the cognitive state of patients for the clinician. J Psychiatr Res 1975;12:189-98.

21. Hoffman WE, Kochs E, Werner C, et al. Dexmedetomidine improves neurologic outcome from incomplete ischemia in the rat. Reversal by the alpha 2-adrenergic antagonist atipamezole. Anesthesiology 1991;75:328-32.

22. Eser O, Fidan H, Sahin O, et al. The influence of dexmedetomidine on ischemic rat hippocampus. Brain Res 2008;1218:250-6.

23. Srivastava VK, Agrawal S, Kumar S, et al. Comparison of dexmedetomidine, propofol and midazolam for shortterm sedation in postoperatively mechanically ventilated neurosurgical patients. J Clin Diagn Res 2014;8:GC04-7.

24. Yang NL, Xu DM, Ming GF, et al. Intervention Effect of Dexmedetomidine on Inflammatory Response Following Traumatic Brain Injury in Rats. Pract Prev Med 2010;17:243-5.

25. Ueda K, Kaneda Y, Sakano H, et al. Obstacles for shortening hospitalization after video-assisted

Cite this article as: Zhang LY, Zhang YH, Shen J, Luo Y. Effects of dexmedetomidine on post-operative recovery and mental status in patients receiving robotic-assisted thoracic surgery. Ann Palliat Med 2019;8(4):469-475. doi: 10.21037/ apm.2019.08.09 pulmonary resection for lung cancer. Ann Thorac Surg 2003;76:1816-20.

26. Jiang L, Li L, Shen J, et al. Effect of dexmedetomidine on lung ischemiareperfusion injury. Mol Med Rep 2014;9:419-26.

27. Wu CY, Lu YF, Wang ML, et al. Effects of Dexmedetomidine Infusion on Inflammatory Responses and Injury of Lung Tidal Volume Changes during One-Lung Ventilation in Thoracoscopic Surgery: A Randomized Controlled Trial. Mediators Inflamm 2018;2018:2575910.

28. Oxlad M, Stubberfield J, Stuklis R, et al. Psychological risk factors for increased post-operative length of hospital stay following coronary artery bypass graft surgery. J Behav Med 2006;29:179-90.

29. Myles PS, Hunt JO, Fletcher H, et al. Relation between quality of recovery in hospital and quality of life at 3 months after cardiac surgery. Anesthesiology 2001;95:862-7. 